\title{
O Uso da Trajetória de Aprendizagem do Aluno em Ambientes Virtuais de Aprendizagem
}

\author{
Eduardo José de Borba, DCC/UDESC, eduardojoseborba@gmail.com \\ Isabela Gasparini, PPGCA e PPGECMT, DCC/UDESC, isabela.gasparini@udesc.br
}

\begin{abstract}
Resumo: Os Ambientes Virtuais de Aprendizagem produzem quantidades de dados significativas sobre a interação dos alunos com o ambiente. A trajetória da aprendizagem representa o caminho utilizado pelos alunos para alcançar os objetivos educacionais e pode ser obtida pela da análise desses dados. Através da análise da trajetória de aprendizagem, o professor pode retirar importantes informações sobre o comportamento dos alunos. O objetivo desse trabalho é realizar uma fundamentação da trajetória de aprendizagem, desde sua captura até a visualização, e descrever uma proposta de ferramenta para a análise da trajetória. Foram realizados levantamentos sobre as principais técnicas relacionadas à trajetória de aprendizagem e de trabalhos que realizaram esse tipo de análise. O resultado esperado é a criação de uma ferramenta para a captura e visualização de forma automática da trajetória que permita ao professor entender o comportamento de seus alunos.
\end{abstract}

Palavras-chave: trajetória de aprendizagem, ambiente virtual de aprendizagem, captura e visualização.

\section{The Use of Student Learning Trajectory for Virtual Learning Environments}

\begin{abstract}
Virtual Learning Environments create significant amount of data about learners' interaction with the environment. The learning trajectory represents the path used by learners to reach the educational goals and can be obtained by the analysis of this data. Through the analysis of the learning trajectory, the teacher could pull relevant information about the learner's behavior. This paper aims to present the elements of the learning trajectory, from capture to visualization, and to describe a tool's proposed to analyze the learners' trajectory. Were described the main techniques related to learning trajectory and the literature review on the field. As result, we intent to develop a tool to automatic capture and visualize the trajectory, to help teachers understand learner's behavior.
\end{abstract}

Keywords: learning trajectory, virtual learning environment, capture and visualization.

\section{INTRODUÇÃ̃O}

Os Ambientes Virtuais de Aprendizagem (AVAs) são ambientes computacionais com a finalidade de integrar diversas mídias (e.g, vídeos, apresentações, textos) e dar suporte à educação online (Ferro et al., 2011). Eles surgiram para permitir que estudantes e professores utilizem recursos computacionais e a internet com o objetivo de facilitar o 
processo de ensino-aprendizagem. $O$ uso desses ambientes vem aumentando consideravelmente nos últimos anos e os AVAs geram diariamente uma quantidade enorme de dados sobre os alunos, suas interações com o ambiente, sobre os professores, suas disciplinas, etc. Esses dados podem possuir informações importantes a serem analisadas para auxiliar no processo de ensino-aprendizagem.

A trajetória de aprendizagem é uma importante análise que pode ser realizada sobre esses dados. Ela pode auxiliar tanto o sistema como o professor a extrair informações sobre o comportamento do aluno através da forma como ele acessa o ambiente. Para o sistema, a trajetória pode auxiliar na identificação do perfil do usuário, seu estilo cognitivo de aprendizagem, suas preferências, etc. Para o professor, a trajetória pode ajudá-lo a identificar problemas na aprendizagem dos alunos, verificar se os alunos realizaram as tarefas planejadas, se os alunos estão acessando sua disciplina da maneira esperada, etc.

O objetivo deste trabalho é realizar uma fundamentação sobre a Trajetória de Aprendizagem, levantando as principais técnicas de captura, análise e visualização da informação (seção 2), um levantamento de trabalhos que exploram a trajetória de maneiras distintas (seção 3) e a descrição de uma proposta de ferramenta para a captura e visualização da trajetória (seção 4). Por último, é apresentada a conclusão (seção 5) e as referências bibliográficas.

\section{FUNDAMENTOS}

Nesta seção são apresentados os principais conceitos envolvendo a Trajetória de Aprendizagem, sua captura, análise e aplicação em AVAs.

A trajetória de aprendizagem está relacionada ao caminho que o aluno percorre dentro de uma determinada disciplina num AVA (Schoonenboom et al., 2007). O "caminho é o conjunto de passos dado pelo usuário ao interagir com o ambiente" (Palazzo et al., 2014).

A trajetória de aprendizagem percorrida pelo aluno pode conter informações importantes para serem utilizadas tanto pelo AVA quanto pelo professor. Para o professor a importância da trajetória está relacionada a identificar se a maioria dos alunos está percorrendo os objetos de aprendizagem na ordem desejada ou planejada (Fortenbacher et al., 2013). Além disso, a trajetória de aprendizagem pode auxiliar o professor a compreender o comportamento do aluno e a identificar possíveis problemas em suas disciplinas (d'Aquin e Jay, 2013). Já para o AVA, capturar a trajetória de aprendizagem do aluno pode auxiliar em diversas tarefas, tais como na recomendação de materiais (Salehi et al., 2014), na identificação de dependências entre conceitos de uma matéria (Aoki et al., 2013), na atualização do perfil do usuário (Mosharraf et al., 2013), entre outras.

As técnicas de Web Analytics são essenciais para a captura da Trajetória de Aprendizagem do aluno, principalmente na etapa da extração de dados. Web Analytics é um processo que envolve desde a coleta, medição, monitoramento e análise dos dados relacionados ao acesso às páginas web, até a elaboração de relatórios para entender a experiência dos usuários no sistema (Hasan et al., 2009). As duas principais técnicas de 
coleta de dados em Web Analytics são através de Web Logs e através de Page Tagging (Beasley, 2013).

$\mathrm{Na}$ técnica de Web Logs as análises são realizadas em arquivos de $\log$ que armazenam as transações de um servidor $W e b$. Toda vez que uma requisição é enviada ao servidor ela é armazenada nesse arquivo de $\log$ (Beasley, 2013). Esses arquivos de Logs armazenam algumas informações básicas como endereço IP, data e hora da requisição, página ou arquivo requisitado, origem da requisição e informações do navegador e sistema operacional do usuário (Jansen, 2009).

O Page Tagging é uma técnica que consiste em adicionar um pequeno trecho de código em JavaScript nas páginas do site para rastrear o usuário e capturar as informações que desejar (Beasley, 2013). Quando o navegador do usuário carrega uma página, ele abre o html e começa a interpretar os códigos JavaScript. Ao interpretar o código referente ao Page Tagging, o navegador irá automaticamente enviar algumas informações para o banco de dados da aplicação, por exemplo (Beasley, 2013): que página foi carregada, quando foi carregada, de onde o usuário veio, endereço IP, detalhes técnicos (navegador, sistema operacional, resolução da tela, etc.).

A vantagem da técnica de Web Logs é que a captura é feita do lado do servidor, enquanto o Page Tagging é executado no lado do cliente e necessita que o JavaScript do navegador esteja habilitado (Beasley, 2013). Por outro lado, a abordagem de Page Tagging fornece dados com maior precisão e mais completos para as aplicações de $W e b$ Analytics do que a Web Logs (Hasan et al., 2009), além do Page Tagging ser mais comum nas soluções comerciais (Clifton, 2008) e dos dados gerados através dessa técnica serem mais fáceis de analisar do que da abordagem de Web Logs (Beasley, 2013).

Após a captura da trajetória de aprendizagem, várias análises podem ser realizadas. Uma análise comumente realizada é a identificação dos caminhos mais frequentes (Özyer et al., 2004; Salehi et al., 2014). Esse tipo de análise pode servir para diversos propósitos, como: a recomendação de itens/materiais a um estudante, entender o comportamento do aluno em uma disciplina ou a própria avaliação do ambiente e da sua interface. Essas análises utilizam técnicas de Sequential Pattern, que são técnicas de identificação de padrões para sequências de informações. Segundo Nguyen e Do (2009) as técnicas de Sequential Pattern se dividem em duas abordagens: Candidate Generation-and-test e Pattern-grownth.

A abordagem Candidate Generation-and-test é uma extensão do algoritmo de descobrimento de regras de associação Apriori, satisfazendo a premissa "toda subsequência não-vazia de uma sequência frequente como uma sequência frequente" (Nguyen e Do, 2009). Alguns exemplos de algoritmos dessa abordagem são: AprioriAll, GSP e SPADE.

Já na abordagem Pattern-grownth a mineração é realizada separadamente para cada sequência frequente com bancos de dados disjuntos com o propósito de reduzir o número de elementos (Nguyen e Do, 2009). Um exemplo dessa abordagem é o algoritmo FreeSpan.

Outra análise é a comparação com um caminho considerado padrão (aquela trajetória definida pelo professor como a melhor estratégia para o processo de ensino- 
aprendizagem) (Bezdan et al., 2013; Ceddia et al., 2007). Esse tipo de comparação pode servir para identificar se determinadas atividades foram realizadas e se foram realizadas de forma correta. A trajetória também pode auxiliar na validação da interface de um ambiente. Bezdan et al. (2013) descreve um método para realizar esse tipo de análise. Em seu trabalho as sequências de páginas acessadas são representadas através de uma cadeia de caracteres (string), onde cada letra representa uma página distinta. Através da distância de Levenshtein são identificados quais caminhos estão mais próximos da string padrão e quais estão muito diferentes. Quanto mais próxima a string estiver da original, mais próxima é a trajetória de aprendizagem do aluno com o planejado pelo professor.

$\mathrm{Na}$ seção 3 são apresentados alguns trabalhos relacionados que utilizam a trajetória de aprendizagem em aplicações educacionais com diferentes propósitos. Esses trabalhos apresentam a técnica utilizada para capturar os dados, quais análises foram realizadas e qual a implicação para o processo de ensino-aprendizagem.

\section{TRABALHOS RELACIONADOS}

O objetivo desta seção é fazer o levantamento de trabalhos sobre trajetórias de aprendizagem. Foram levantados cinco trabalhos: d'Aquin e Jay (2013); Ceddia et al. (2007); Salehi et al. (2014); Fortenbacher et al. (2013); e Palazzo et al. (2014). Esses cinco trabalhos exploram a trajetória de aprendizagem de maneira bem distintas e serão explicados em mais detalhes nos próximos parágrafos.

O trabalho de d'Aquin e Jay (2013) propõe o uso de algoritmos de Sequential Pattern combinados com Linked data para melhorar o processo de interpretação das interações do aluno. Foram utilizados os dados armazenados no servidor web ( $\mathrm{Web}$ Logs) para realizar as análises e identificar os caminhos que são mais frequentes no ambiente. Essa análise foi realizada utilizando o algoritmo PrefixSpan. Segundo Heth e Bizer (2011), os Linked data são um conjunto de princípios e tecnologias que dependem da arquitetura da internet (links) para compartilhar, modelar e integrar os dados. Esse trabalho combinou então as análises da trajetória de aprendizagem com os dados obtidos através de Linked data. Como resultado, o trabalho apresentou análises mais precisas sobre os caminhos percorridos, permitindo analisar, além da sequência de páginas acessadas, informações pertinentes sobre cada uma dessas páginas para entender o comportamento do aluno.

Com o objetivo de entender o comportamento do aluno, o trabalho de Ceddia et al. (2007) descreve uma ferramenta para análise das interações através dos arquivos de Log capturados. A ferramenta, chamada WAT (Weblog Analysis Tool), permite que o educador descreva sequências de atividades que são interessantes no contexto do curso e, ao processar os arquivos de $\log$, a ferramenta irá determinar que usuários realizaram tais sequências.

O autor define atividades de aprendizagem como sendo uma série de interações com um website para alcançar um resultado particular e divide as atividades em cinco categorias hierárquicas: apresentação, organizacional, exploratória, focadas em objetivo e complexas (Ceddia et al., 2007). A primeira categoria (apresentação) faz preparação 
para alguma tarefa, e ao aumentar o nível na hierarquia, as atividades ficam mais complexas e completas, sendo dos últimos níveis as tarefas essenciais para o aprendizado. A abstração das interações do usuário em atividades de aprendizagem permite que a ferramenta realize análises nos arquivos de $\log$ e obtenha informações sobre o usuário e as tarefas que ele estava realizando. Para validar a ferramenta desenvolvida, os autores realizaram um estudo de caso com os arquivos de Log de um website chamado WIER, que possui predominantemente atividades do tipo focadas em objetivos, e várias informações sobre as interações dos usuários são exploradas.

Primo et al. (2010) afirmou que devemos ter mais cuidado ao desenvolver um sistema de recomendação para um ambiente educacional, pois uma má sugestão de um filme (no Netflix, por exemplo) é muito menos "doloroso" ao usuário do que receber uma sugestão de algum conteúdo educacional que o desmotive a aprender. O trabalho de Salehi et al. (2014) considera, entre outras características, os caminhos percorridos pelos alunos no ambiente no momento de realizar as recomendações de materiais ao aluno. Os autores combinaram a filtragem colaborativa, uma técnica de sistemas de recomendação que considera usuários com gostos semelhantes para identificar os materiais mais interessantes ao usuário, com as informações extraídas das interações desse usuário para realizar uma recomendação mais precisa. Salehi et al. (2014) afirma que existe uma ordem intrínseca para os materiais de aprendizagem no processo de aprendizagem de um aluno, por exemplo: o aluno provavelmente irá aprender do material mais fácil para o mais difícil, do mais teórico para o mais prático, etc. Com base nisso, a mineração dos materiais acessados pelo aluno e a ordem acessada ajudarão a prever o que ele irá precisar no futuro. São utilizados dois algoritmos de Sequential Pattern para a construção da árvore de preferência do aluno (árvore com os caminhos mais frequentes): Apriori e PrefixSpan.

Fortenbacher et al. (2013) descreve uma ferramenta de Web Analytics para AVAs chamada LeMo. Entre outras análises realizadas por essa ferramenta, ela realiza a análise das trajetórias de aprendizagem. A ferramenta LeMo permite a conectividade com três diferentes plataformas: Moodle, Clix e Chemgapedia. Para o Moodle e o Clix a ferramenta LeMo possui conectores implementados que acessam diretamente o banco de dados das aplicações. Para o Chemgapedia, que é uma aplicação web, os dados são extraídos dos arquivos de $\log$. A identificação dos caminhos mais frequentes é realizada através do algoritmo BIDE, que utiliza uma abordagem Apriori.

O grande diferencial do trabalho de Fortenbacher et al. (2013) está em realizar e descrever a visualização da trajetória de aprendizagem. A Visualização da Informação é uma área que combina características de Computação Gráfica com outras áreas da computação para realizar representações manipuláveis de grandes quantidades de dados, transformando os dados em informações significativas (Gherson et al., 1998). O LeMo permite tipos diferentes de visualização da trajetória, tais como o grafo de atividade, representado através de uma rede de navegação, ou dos caminhos mais frequentes, sendo os nós os objetos de aprendizagem e as arestas os passos de navegação. Essas visualizações permitem ao responsável pela disciplina, normalmente o professor, realizar análises com relação aos caminhos percorridos para entender melhor o comportamento dos alunos e como a sua disciplina está sendo acessada. 
O último trabalho relacionado é o de Palazzo et al. (2014). Com o objetivo de contribuir para a inclusão digital e permitir o desenvolvimento de um ambiente sensível às características geoculturais dos alunos, esse trabalho realizou uma análise da interação dos alunos de diferentes regiões com o mesmo ambiente. Essa análise visava descobrir os padrões de acesso dos alunos. Foram realizados experimentos com alunos de três países (Brasil, Argentina e Uruguai) em um curso sobre "Riscos e Danos da Internet" do ambiente Moodle e os $\operatorname{Logs}$ foram armazenados e analisados posteriormente. Com base nessas análises foram geradas visualizações dos padrões navegacionais dos alunos dos diferentes países. Os padrões eram bem diferentes entre os três grupos, por exemplo: no grupo brasileiro houve muitos saltos não sequenciais, indo direto para os questionários e voltando para o assunto para encontrar as respostas, enquanto no grupo argentino os alunos seguiram mais sequencialmente no curso.

Essas análises realizadas por Palazzo et al. (2014) são muito interessantes pois, com base nas visualizações geradas das interações dos alunos, podemos retirar informações importantes sobre o seu comportamento, sobre a forma como utilizam o ambiente e sobre os diferentes estilos de aprendizagem. Porém, essas visualizações não são geradas de forma automática, elas foram geradas off-line após os experimentos terem sido realizados. Para um professor, seria importante ter essas informações em tempo real, geradas automaticamente pelo ambiente e lhe dando suporte para ministrar sua disciplina. Dos trabalhos de d'Aquin e Jay (2013), Ceddia et al. (2007) e Salehi et al (2014) pudemos obter importantes informações sobre algoritmos de análises de logs para a identificação da trajetória de aprendizagem. Enquanto do trabalho de Fortenbacher et al. (2013), pudemos entender melhor a visualização dos grafos de navegação dos alunos.

Na próxima seção descreveremos uma proposta de ferramenta para a análise da trajetória de aprendizagem no ambiente AdaptWeb ${ }^{\circledR}$. O objetivo desta ferramenta é gerar visualizações de maneira automática para auxiliar o professor em sua disciplina.

\section{ANÁlISE DA TRAJETÓRIA DE APRENDIZAGEM NO AMBIENTE ADAPTWEB ${ }^{\circledR}$}

O AdaptWeb ${ }^{\circledR}$ (Ambiente de Ensino-Aprendizagem Adaptativo na Web) é um sistema open source que consiste em um AVA capaz de adaptar o conteúdo, a apresentação e a navegação em determinado curso às características e preferências do aluno (Gasparini et al., 2009).

Este sistema possui dois ambientes: o de autoria e o do aluno. No ambiente de autoria o professor cria e organiza a estrutura dos conteúdos de sua disciplina. Essas disciplinas podem ser ministradas em mais de um curso, e.g., a disciplina de Cálculo pode ser oferecida nos cursos de Ciência da Computação, Engenharia Elétrica e Engenharia Civil, com o conteúdo devidamente adaptado para cada curso. O ambiente do aluno é organizado em três seções: o Ambiente de Aula, onde o aluno vê o conteúdo da disciplina; Fórum de Discussão, que permite que os usuários interajam entre si; Mural de Recados, onde o aluno pode visualizar e enviar mensagens. 
No AdaptWeb ${ }^{\circledR}$, cada tópico do conteúdo programático da disciplina pode ser divido em quatro categorias: Conceito, Exemplos, Exercícios e Material Complementar. $\mathrm{Na}$ categoria Conceito o professor costuma colocar os conceitos principais daquele tópico. $\mathrm{Na}$ categoria Exemplos podem conter exemplos que ajudem a compreender melhor o assunto. Na categoria Exercícios aparecem exercícios sobre o tópico com o objetivo de testar ou de apenas exercitar o conteúdo. Já na categoria Material Complementar os professores costumam colocar vários objetos de aprendizagem que podem auxiliar o aluno no processo de ensino-aprendizagem, desde links de sites até artigos, vídeos, imagens, etc.

Para auxiliar o professor a compreender o comportamento dos alunos no ambiente AdaptWeb ${ }^{\circledR}$, foi implementada uma ferramenta Web Analytics. Os dados são coletados nas três seções que o aluno pode acessar e, a partir dos dados coletados, algumas métricas são calculadas. Estas métricas foram divididas em quatro categorias, de modo a facilitar a compreensão do professor, e são:

- Ambiente de Aula: Métricas relacionadas ao Ambiente de Aula, i.e., Modo de Navegação, Total de Usos do Sistema de Busca, Palavras-chave Pesquisadas, Total de Acessos aos Conceitos, Total de Acessos aos Exercícios, Total de Acessos aos Exemplos e Total de Acessos aos Materiais Complementares;

- Fórum de Discussão: Métricas relacionadas a utilização do Fórum de Discussão, i.e., Total de Acessos aos Tópicos, Total de Tópicos Criados e Total de Respostas;

- Mural de Recados: Métricas para analisar a troca de recados dos alunos, i.e., Total de Recados Enviados, Tipos de Recados Enviados (para os outros alunos ou para o professor) e Total de Visualizações;

- Uso geral: Métricas não relacionadas às três seções principais, i.e., Total de Visitas por Aluno, Tempo Médio de Acesso dos Alunos, Frequência de Acesso, Total de Acessos a Cada Seção, Sistema Operacional, Navegadores e Resoluções de Tela.

Os dados referentes a cada uma das métricas calculadas pela ferramenta $\mathrm{Web}$ Analytics passam por um processo de transformação, para então serem geradas as representações que serão apresentadas ao professor. As técnicas de visualização da informação permitem ao usuário compreender as informações com mais facilidade (Freitas et al., 2001) e por isso foram escolhidas para representar essas métricas. A transformação dos dados é realizada com base no modelo de Card et al. (1999).

Utilizando como exemplo a métrica Total de Visitas por Aluno, os dados calculados pela ferramenta Web Analytics são transformados em uma estrutura de dados similar a uma tabela, onde cada aluno possui um valor que representa o seu número de acessos. A partir desta estrutura, cada aluno se torna uma barra no gráfico de barras, cujo tamanho representa o total de visitas. Ao exibir o gráfico resultante para o professor ele pode alterar algumas propriedades do gráfico ou dos dados para melhorar a sua compreensão sobre o conjunto de dados em análise.

Um importante recurso que não é analisado por esta ferramenta de Web Analytics é a trajetória de aprendizagem. O ambiente AdaptWeb ${ }^{\circledR}$ já possui as 
ferramentas necessárias para captura e análises mais simples com base em arquivos de Log, bem como a visualização de informações. A partir disso, propõe-se acrescentar à ferramenta de Web Analytics um algoritmo de análise de Logs que permitirá aos professores compreender como os alunos acessam sua disciplina. Esse algoritmo, como levantado nas pesquisas bibliográficas, é um algoritmo de Sequential Pattern, que identifica os caminhos mais frequentes realizados no ambiente, podendo ser tanto da abordagem Pattern Growth como da abordagem Candidate Generation-and-Test. Após analisados os Logs, o resultado desse algoritmo poderá ser visualizado pelo professor através da ferramenta de visualização. Uma estratégia bastante utilizada para a visualização da trajetória de aprendizagem é através de grafos, onde os nós representam as páginas e as arestas representam a navegação dos alunos.

Este trabalho irá estender a ferramenta de Web Analytics e visualização da informação, incorporando a análise da trajetória de aprendizagem do aluno em cada disciplina. Desta forma, o professor poderá visualizar o percurso de cada aluno, por quais tópicos e categorias (conceito, exemplo, exercício e material complementar) o aluno navega, se o aluno explora a disciplina de uma maneira mais sequencial/linear, ou assiste a disciplina de forma exploratória, comparar os diferentes caminhos percorridos por cada aluno em uma mesma disciplina, observar a quantidade de alunos que necessitou de auxílio ou utilizou mecanismos de navegação como suporte ao entendimento da disciplina, etc.

\section{CONCLUSÃO}

Nesse trabalho foi primeiramente realizado um levantamento dos fundamentos necessários à compreensão da Trajetória da Aprendizagem. A Trajetória de Aprendizagem representa o caminho que um aluno ou um grupo de alunos percorreu no ambiente de aprendizagem. A Trajetória é obtida através da captura dos dados gerados com a interação do aluno com o ambiente. Essa captura pode ser realizada através de duas técnicas de Web Analytics: Page Tagging, onde o navegador do aluno executa scripts embutidos na página e manda informações ao banco de dados da aplicação; Web Logs, onde são analisados os Logs de acesso.

Existem diversas técnicas para a análise da Trajetória de Aprendizagem do aluno. A principal técnica é a de Sequential Pattern. Essa técnica levanta os caminhos mais frequentes realizados pelos alunos no ambiente e seus algoritmos podem ser divididos em duas categorias: Pattern Grownth, onde a mineração é realizada separadamente para cada sequência com o propósito de reduzir o número de elementos; Candidate Generation-and-Test, que consiste na descoberta de regras de associação e que considera toda subsequência não-vazia de uma sequência frequente como uma sequência frequente.

A Trajetória de Aprendizagem possui diferentes aplicações, como pode ser visto nos trabalhos relacionados. As principais aplicações levantadas foram: entender o comportamento do aluno, identificando sequências de acessos que foram realizadas por estes; recomendação de materiais, onde a trajetória auxilia o sistema a conhecer mais sobre a situação atual do aluno para identificar os materiais mais adequados para ele; visualização da trajetória, onde o professor pode mais facilmente analisar as 
características de acesso dos seus alunos e até mesmo identificar como a cultura pode influenciar a forma como os alunos percorrem as páginas do ambiente.

Foi escolhido o ambiente AdaptWeb ${ }^{\circledR}$ para a implementação de uma ferramenta de captura e visualização da Trajetória de Aprendizagem. Esse ambiente foi escolhido por já possuir uma ferramenta de Web Analytics, para o cálculo de diversas métricas referentes à interação dos alunos, e uma ferramenta de visualização da informação para a melhor compreensão dessas métricas pelo professor. A ferramenta proposta utilizará dados que já são capturados pelo ambiente, aplicando técnicas de análise do caminho e de visualização da informação para que o professor tenha acesso a Trajetória de Aprendizagem de seus alunos.

\section{REFERÊNCIAS BIBLIOGRÁFICAS}

AOKI, S.; SAGA, R.; ICHINOTSUBO, T.; NIU, W.; TSUJI, H. Dependency Extraction from Growth Trajectory using Sequential Pattern. i-Know '13, Graz, Austria, 2013.

BEASLEY, M. Practical Web Analytics for User Experience. USA: Elsevier, 2013.

BEZDAN, E.; KESTER, L.; KIRSCHNER, P. A. The influence of node sequence and extraneous load induced by graphical overviews on hypertext learning. Computers in Human Behavior, vol. 29, 870-880, 2013.

CARD, S. K.; MACKINLAY, J. D.; SHNEIDERMAN, B. Readings in Information Visualization: using vision to think. Morgan Kauffman, 1999.

CEDDIA, J.; SHEARD, J.; TIBBEY, G. WAT- A Tool for Classifying Learning Activities from a Log File. Ninth Australasian Computing Education Conference (ACE2007), Ballarat, Victoria, Australia, 2007.

CLIFTON, B. Advanced Web Metrics with Google Analytics. Indianapolis, Indiana: Wiley Publishing Inc, 2008.

D'AQUIN, M.; JAY, N. Interpreting Data Mining Results with Linked Data for Learning Analytics: Motivation, Case Study and Directions. LAK '13, Leuven, Belgium, 2013.

FERRO, M. R. da C.; NASCIMENTO Jr., H. M.; PARAGUAÇU, F.; COSTA, E. de B; MONTEIRO, L. A. L. Um modelo de sistema de recomendação de materiais didáticos para ambientes virtuais de aprendizagem. Anais do XXII SBIE - XVII WIE, Aracaju. 2011.

FORTENBACHER, A.; BEUSTER, L.; ELKINA, M.; KAPPE, L.; MERCERON, A.; PURSIAN, A.; SCHWARZROCK, S.; WENZLAFF, B. LeMo: a Learning Analytics Application Focussing on User Path Analysis and Interactive Visualization. 7th IEEE International Conference on Intelligent Data Acquisition and Advanced Computing Systems: Technology and Applications, 2013.

FREITAS, C. M. D. S.; CHUBACHI, O. M.; LUZZARDI, P. R. G.; CAVA, R. A. Introdução à Visualização de Informações. Revista de Informática Teórica e Aplicada, Porto Alegre, RS, Brasil, v. 8, n. 2, p. 143-158, 2001. 
GASPARINI, I.; PALAZZO M. de OLIVEIRA, J. ; PIMENTA, M. S.; de LIMA, J. V.; KEMCZINSKI, A.; PROENÇA Jr, M. L.; BRUNETTO, M. A. C. AdaptWeb Evolução e Desafios. Cadernos de Informática (UFRGS), v. 4, p. 47-54, 2009.

GHERSON, N.; EICK, S. G.; CARD, S. Information Visualization. Interactions, New York, NY, USA, v. 5, n. 2: 9-15, 1998.

HASAN, L.; MORRIS, A.; PROBETS, S. Using Google Analytics to Evaluate the Usability of E-commerce Sites. Congresso Human Centered Design, HCII 2009, San Diego, EUA, 2009.

HEATH, T; BIZER, C. Linked Data: Evolving the Web into a Global Data Space. Synthesis Lectures on the Semantic Web: Theory and Technology, Morgan \& Claypool, 2011.

JANSEN, B. J. Understanding User-Web Interactions via Web Analytics. Morgan \& Claypool, 2009.

MOSHARRAF, M.; TAGHIYAREH, F.; KHARRAT, M. Equipping Children eLearning Systems with a Hybrid Personality Type Indicator. 4th International Conference on e-Learning and e-Teaching (ICELET), 2013.

NGUYEN, L.; DO, P. Learning concept recommendation based on sequential pattern mining. 3rd IEEE international conference on digital ecosystems and technologies, 2009.

ÖZYER, T.; ALHAJJ, R.; BARKER, K. Multi-Dimensional Sequential Web Mining by Utilizing Fuzzy Inferencing. International Conference on Machine Learning and Applications, 2004.

PALAZZO M. de OLIVEIRA, J.; VALDENI de LIMA, J.; KRUG, L.; PERNAS, A. M.; GASPARINI, I.; FERNÁNDEZ, A.; DÍAZ, A. Adaptatividade geocultural em ambientes virtuais de aprendizagem. Revista Iberoamericana de Educación a Distancia (RIED), v. 17: 1, pp 83-109, 2014.

PRIMO, T. T.; VICARI, R. M.; DA SILVA, J. M. C. Rumo ao uso de metadados educacionais em sistemas de recomendação. Simpósio Brasileiro de Informática na Educação (SBIE). 2010.

SALEHI, M.; KAMALABADI, I. N.; GHOUSHCHI, M. B. G. Personalized recommendation of learning material using sequential pattern mining and attribute based collaborative filtering. Educ Inf Technol, 19:713-735, 2014.

SCHOONENBOOM, J.; LEVENE, M.; HELLER, J.; KEENOY, K.; TURCSANYISZABO, M. Trails in Education: Technologies that Support Navigational Learning. Sense Publishers, 2007. 